\title{
"Testing of weak form of efficient market hypothesis: evidence from the Bahrain Bourse"
}

\begin{tabular}{|c|c|}
\hline AUTHORS & $\begin{array}{l}\text { lqbal Thonse Hawaldar } \mathbb{D} \text { https://orcid.org/0000-0001-7181-2493 } \\
\text { R https://publons.com/researcher/1456475/iqbal-thonse-hawaldar/ } \\
\text { Babitha Rohit } \\
\text { Prakash Pinto (D) https://orcid.org/0000-0001-8168-9679 }\end{array}$ \\
\hline ARTICLE INFO & $\begin{array}{l}\text { Iqbal Thonse Hawaldar, Babitha Rohit and Prakash Pinto (2017). Testing of } \\
\text { weak form of efficient market hypothesis: evidence from the Bahrain Bourse. } \\
\text { Investment Management and Financial Innovations, 14(2-2), 376-385. } \\
\text { doi:10.21511/imfi.14(2-2).2017.09 }\end{array}$ \\
\hline DOI & http://dx.doi.org/10.21511/imfi.14(2-2).2017.09 \\
\hline RELEASED ON & Monday, 21 August 2017 \\
\hline RECEIVED ON & Sunday, 02 April 2017 \\
\hline ACCEPTED ON & Tuesday, 11 July 2017 \\
\hline LICENSE & $\begin{array}{l}(c) \text { EY-NC } \\
\text { This work is licensed under a Creative Commons Attribution-NonCommercial } 4.0 \\
\text { International License }\end{array}$ \\
\hline JOURNAL & "Investment Management and Financial Innovations" \\
\hline ISSN PRINT & $1810-4967$ \\
\hline ISSN ONLINE & $1812-9358$ \\
\hline PUBLISHER & LLC "Consulting Publishing Company "Business Perspectives" \\
\hline FOUNDER & LLC "Consulting Publishing Company "Business Perspectives" \\
\hline
\end{tabular}

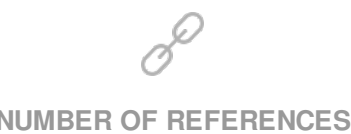

29

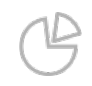

NUMBER OF FIGURES

0

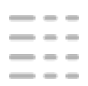

NUMBER OF TABLES

4

(C) The author(s) 2022. This publication is an open access article. 


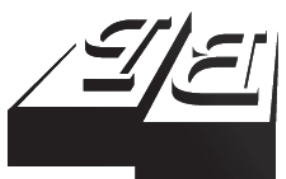

BUSINESS PERSPECTIVES

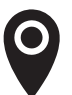

LLC "CPC "Business Perspectives" Hryhorii Skovoroda lane, 10, Sumy, 40022, Ukraine

www.businessperspectives.org

Received on: $2^{\text {nd }}$ of April, 2017 Accepted on: $11^{\text {th }}$ of July, 2017

(C) Iqbal Thonse Hawaldar, Babitha Rohit, Prakash Pinto, 2017

Iqbal Thonse Hawaldar, Dr., Associate Professor, Department of Accounting and Finance, College of Business Administration \& Assistant to the President for Accreditation and Quality Assurance, Kingdom University, Bahrain.

Babitha Rohit, Assistant Professor, Department of Business Administration, St. Joseph Engineering College, India.

Prakash Pinto, Dr., Professor and Dean, Department of Business Administration, St. Joseph Engineering College, India.

\section{(ㅇ)(1) $(8)$}

This is an Open Access article, distributed under the terms of the Creative Commons Attribution-NonCommercial 4.0 International license, which permits re-use, distribution, and reproduction, provided the materials aren't used for commercial purposes and the original work is properly cited.
Iqbal Thonse Hawaldar (Bahrain), Babitha Rohit (India), Prakash Pinto (India)

\section{TESTING OF WEAK FORM OF EFFICIENT MARKET HYPOTHESIS: EVIDENCE FROM THE BAHRAIN BOURSE}

\begin{abstract}
Efficient market hypothesis (EMH) states that financial markets are "informationally efficient", implying that current prices fully reflect all available information. The present study aims at testing the weak form of market efficiency of the individual stocks listed on the Bahrain Bourse for the period 2011 to 2015. Weak form of EMH is tested using the Kolmogorov-Smirnov goodness of fit test, run test and autocorrelation test. The K-S test result concludes that in general the stock price movement does not follow random walk. The results of the runs test reveals that share prices of seven companies do not follow random walk. Autocorrelation tests reveal that share prices exhibit low to moderate correlation varying from negative to positive values. As the study shows mixed results, it is difficult to conclude the weak form of efficiency of Bahrain Bourse.
\end{abstract}

\section{Keywords}

JEL Classification random walk, efficient market hypothesis, run test, autocorrelation test

G100, G140

\section{INTRODUCTION}

Efficient market hypothesis (EMH) states that financial markets are "informationally efficient", implying that current prices fully reflect all available information. According to Fama (1970), there are three forms of EMH. They are: "weak", "semi-strong" and "strong". Weak form states that prices already reflect all past publicly available information. Semi-strong EMH states that price changes reflect past information, as well as reflect new public information. Strong EMH claims that prices instantly reflect even hidden or "insider" information.

The present study tests the weak form of EMH of the individual stocks listed on the Bahrain Bourse for the period 2011 to 2015. Bahrain Stock Exchange established in 1987, officially began operations in 1989, with 29 listed companies and a manual trading system. However, in 2010, Bahrain Bourse (BHB) was established as a shareholding company to replace Bahrain Stock Exchange. The market capitalization of the Bourse at the end of 2015 was BD 7, 199, 907, 825 with 46 listed companies. The Bahrain All-Share Index lists shares under six sectoral indices: commercial banks, investment, insurance, services, industrial and hotels and tourism. 


\section{LITERATURE REVIEW}

A study into the literature reveals large number of publications having examined the existence of the EMH theory in various developed and undeveloped markets with varying results (Ananzeh, 2014). Iqbal and Mallikarjunappa (2008, 2010, 2011) studied on Indian Stock Market and found that Indian Stock Market is not efficient in weak and semi-strong form.

Awan and Subayyal (2016) studied six stock exchanges in Gulf region (Oman, UAE, Kuwait, Saudi Arabia, Bahrain and Qatar) for the period 2011 to 2015. The results provide evidence that the stock prices at the Gulf markets do not follow the random walk model. Asiri (2008) measured the performance of the Bahrain Stock Exchange (BSE) by applying the Dickey Fuller unit root tests, the ARIMA model, and exponential smoothing techniques.

Dahel and Laabas (1999) compared the stock markets of Bahrain, Kuwait, Saudi Arabia and Oman. Rao and Shankaraiah (2003) investigated the stock market efficiency and suggested strategies for Gulf Co-operation Council (GCC) stock markets. They concluded that these markets were neither developed nor informationally efficient and recommended that there must be better networking between these markets.

Sharma (2005) tested the daily returns series of Gulf Co-operation Council (GCC) stock markets and reported that the returns follow a normal distribution.

Elango and Hussein (2007) examined market efficiency across the stock markets in the GCC countries using daily indices data from October 2001 to October 2006. Kolmogorov-Smirnov test has been applied for analysis and the study rejects the null hypothesis that the returns follow a normal distribution for all seven markets. Run test has been used for randomness. It is concluded that the market is not efficient in its weak form.

Very few studies (Solnik, 1973; Ang \& Pohlman, 1978; Buttler \& Malaikah, 1992; Rao \& Shankaraiah, 2003; Moustafa, 2004) have tested the efficiency of individual stocks. Using serial correlation test, Solnik (1973) investigated market efficiency for 234 securities in eight major European stock markets using daily prices.

Ang and Pohlman (1978) examined 54 stocks of five stock exchanges of Japan, Singapore, Australia, Hong Kong and the Philippines and concluded that the markets are slightly efficient in the weakest form.

Moustafa (2004) analyzed the weak form efficiency of United Arab Emirates (UAE) stock market by observing the behavior of stock prices. Three years data were considered from October 2, 2001 to September 1, 2003. Using nonparametric run test on 43 stocks of the Emirates market and the study concluded that stocks didn't follow the normal distribution.

\section{RESEARCH GAP}

There have been very few studies (Gharaibeh \& Hammadi, 2013; Hawaldar, 2015; Hawaldar 2016a; Hawaldar, 2016b; Hawaldar, Shakila, \& Pinto, 2017) on Bahrain Bourse. This has lead the researchers to undertake the present study and contribute to the existing literature on weak form of market efficiency of Bahrain Bourse. The study tests the weak form efficiency of the EMH for individual securities listed on the Bahrain Bourse using run test, Kolmogorov-Smirnov test and autocorrelation test.

\section{OBJECTIVES}

\section{OF THE STUDY}

Based on the review of literature, the following objectives are set and hypotheses are developed to fill the research gap in Bahrain Bourse.

1. To test the weak form efficiency of individual stocks listed on Bahrain Bourse.

2. To examine the randomness of individual stock prices.

3. To test the independence of individual stock price changes. 


\section{Hypotheses}

H01: The individual stock prices follow a random walk.

Ha1: The individual stock prices do not follow a random walk.

H02: The individual stock prices are efficient in weak form of efficient market hypothesis.

Ha2: The individual stock prices are not efficient in weak form of efficient market hypothesis.

H03: The price changes in individual stocks are independent.

Ha3: The price changes in individual stocks are not independent.

\section{METHODOLOGY}

The present study uses daily prices of the companies listed in the Bahrain All Share Index. The period of study is from 2011 to 2015. The sample consists of 43 companies listed on the Bahrain Bourse for which data are available.

The stock returns are defined as follows:

$$
R_{t}=\frac{\log _{p t}}{\log _{p t-1}},
$$

where $R_{t}$ is the return at time $t, \log _{p t}$ is the logarithmic price at time $t$ and $\log _{p t-1}$ is the logarithmic at time $t-1$.

The study analyzes and tests the weak form of efficiency for the individual stock movements. Weak form of EMH is tested using the run test, Kolmogrov-Smirnov goodness of fit test and autocorrelation test.

Run test: run test is a nonparametric test. It is a statistical procedure that examines whether a string of data is occurring randomly given a specific distribution (Pandey, 2003; Elango \& Hussein, 2007; Omar et al., 2013). The run test analyzes the occurrence of similar events that are separated by events that are different. The mean and variance is calculated as follows:

$$
\begin{gathered}
\mu=\frac{2 n_{1} \cdot n_{2}}{n_{1}+n_{2}}+1 . \\
\sigma^{2}=\frac{2 n_{1} \cdot n_{2} \cdot\left(2 n_{1} \cdot n_{2}-n_{1}-n_{2}\right)}{\left(n_{1}+n_{2}\right)^{2} \cdot\left(n_{1}+n_{2}-1\right)} .
\end{gathered}
$$

The standard normal Z-statistic used to conduct a run test is given by:

$$
Z=\frac{\text { Observed }- \text { Expected }}{\sqrt{\text { Variance }}} .
$$

Kolmogrov-Smirnov goodness of fit test (K-S test): the K-S test is a nonparametric test and is used to determine how well a random sample of data fits a particular distribution (Mobareket et al., 2008; Salam, 2013).

$$
\max _{x}\left|F(x)-S_{n}(x)\right| \leq D_{n, \alpha} .
$$

Autocorrelation: Autocorrelation is defined as a mathematical representation of the degree of similarity between a given time series and a lagged version of itself over successive time intervals (Hamid et al., 2010). A higher value of autocorrelation indicates greater correlation in values. Fama (1970) proposes that by using a less restrictive condition of submartingale sequence, low serial correlation may persist in an efficient market.

$$
L B=n \cdot(n+2) \sum_{k-1}^{m} \frac{P^{2 k}}{n-k} \sim \chi^{2},
$$

where $P^{k}$ - auto-correlation coefficients at lagk, $n$-sample size.

Descriptive statistics of the returns of the listed companies on the Bahrain Stock Exchange is presented in Table 1. Delmon Poultry Co. (POLTRY) records the highest mean returns of $1.528 \%$. The returns of companies that have thin trades are GLOBAL, INVCORP, TAKAFUL, UFC and UPI. Bahrain Telecommunication Co. (BATELCO) having the highest number of trading days has a mean return of $-0.057 \%$ with standard deviation of $1.272 \%$. The Bahrain All Share Index records a mean return of $-0.013 \%$ and standard deviation of $0.455 \%$. The results for GLOBAL, TAKAFUL and UPI are not arrived at due to lack of data. 
Table 1. Descriptive statistics of stock returns

Source: authors' calculations.

\begin{tabular}{|c|c|c|c|c|c|}
\hline Company & $\mathbf{N}$ & Mean (\%) & Std. deviation (\%) & Minimum (\%) & Maximum (\%) \\
\hline $\mathrm{ABC}$ & 182 & 0.023 & 3.949 & -10.54 & 9.53 \\
\hline AHLIA & 79 & -0.646 & 4.717 & -29.95 & 9.19 \\
\hline $\mathrm{ALBH}$ & 808 & -0.071 & 1.840 & -10.44 & 9.49 \\
\hline ARIG & 220 & -0.030 & 3.652 & -10.54 & 9.53 \\
\hline$A \cup B$ & 956 & 0.063 & 1.937 & -10.54 & 9.29 \\
\hline BANADER & 70 & -0.212 & 3.433 & -10.38 & 15.63 \\
\hline BARKA & 473 & -0.161 & 2.634 & -14.95 & 9.53 \\
\hline BASREC & 59 & 0.280 & 4.983 & -10.29 & 9.53 \\
\hline BATELCO & 1014 & -0.057 & 1.272 & -12.61 & 6.9 \\
\hline BBK & 551 & 0.027 & 1.452 & -9.53 & 6.12 \\
\hline BCFC & 268 & 0.192 & 2.403 & -9.96 & 8.96 \\
\hline BFM & 48 & -0.112 & 3.197 & -9.98 & 9.53 \\
\hline BHOTEL & 117 & 0.222 & 3.857 & -13.44 & 9.53 \\
\hline BISB & 396 & -0.068 & 2.942 & -10.54 & 9.53 \\
\hline BKIC & 69 & 0.138 & 3.981 & -10.44 & 9.53 \\
\hline $\mathrm{BMB}$ & 25 & -3.423 & 8.619 & -21.94 & 9.53 \\
\hline BMMI & 326 & 0.072 & 2.204 & -19.53 & 8.91 \\
\hline $\mathrm{BHN}$ & 100 & -0.062 & 5.412 & -10.82 & 9.33 \\
\hline BTC & 121 & -0.304 & 3.392 & -10.38 & 9.19 \\
\hline CINEMA & 79 & 0.885 & 4.354 & -18.19 & 9.53 \\
\hline CPARK & 66 & 0.073 & 5.531 & -10.54 & 9.53 \\
\hline DUTYF & 387 & 0.021 & 2.708 & -34.79 & 9.35 \\
\hline ESTERAD & 359 & -0.103 & 3.048 & -10.54 & 9.53 \\
\hline FAMILY & 34 & 0.025 & 4.217 & -10.05 & 8.34 \\
\hline GFH & 474 & -0.085 & 8.031 & -42.2 & 136.61 \\
\hline GLOBAL & 0 & 0 & 0 & 0 & 0 \\
\hline INOVEST & 195 & -0.546 & 5.914 & -50.46 & 21.87 \\
\hline INVCORP & 6 & -18.125 & 12.730 & -35.67 & -0.02 \\
\hline ITHMR & 842 & -0.043 & 3.538 & -14.66 & 12.26 \\
\hline $\mathrm{KHCB}$ & 753 & -0.146 & 2.336 & -9.68 & 9.53 \\
\hline NASS & 873 & -0.045 & 1.828 & -10.46 & 9.47 \\
\hline NBB & 631 & 0.063 & 2.051 & -10.18 & 9.53 \\
\hline NHOTEL & 32 & -1.232 & 3.911 & -11.44 & 9.07 \\
\hline POLTRY & 26 & 1.528 & 4.290 & -10.54 & 9.37 \\
\hline SALAM & 988 & 0.034 & 2.798 & -10.54 & 12.52 \\
\hline SEEF & 672 & 0.039 & 1.402 & -7.11 & 9.53 \\
\hline TAKAFUL & 1 & -69.31 & 0 & -69.31 & -69.31 \\
\hline TRAFCO & 122 & -0.197 & 2.696 & -9.76 & 9.38 \\
\hline UFC & 2 & 3.9 & 5.515 & 0 & 7.8 \\
\hline UGB & 129 & -0.020 & 4.828 & -10.82 & 9.53 \\
\hline UGIC & 118 & 0.019 & 4.922 & -15.86 & 9.53 \\
\hline UPI & 5 & 0 & 0 & 0 & 0 \\
\hline ZAINABH & 31 & -0.032 & 0.602 & -1.01 & 1.98 \\
\hline Bahrain Index & 1231 & -0.013 & 0.455 & -2.84 & 1.6 \\
\hline
\end{tabular}


Table 2. Results of K-S test

Source: authors' calculations.

\begin{tabular}{|c|c|c|c|c|c|}
\hline \multicolumn{6}{|c|}{ Results of the Kolmogorov-Smirnov test } \\
\hline Company & Z-value & p-value & Company & Z-value & p-value \\
\hline $\mathrm{ABC}$ & 3.377 & $0.000^{*}$ & CPARK & 2.173 & $0.000^{*}$ \\
\hline AHLIA & 2.609 & $0.000^{*}$ & DUTYF & 5.504 & $0.000^{*}$ \\
\hline $\mathrm{ALBH}$ & 7.087 & $0.000^{*}$ & ESTERAD & 5.402 & $0.000^{*}$ \\
\hline ARIG & 4.230 & $0.000^{*}$ & FAMILY & 1.701 & $0.006^{*}$ \\
\hline AUB & 6.487 & $0.000^{*}$ & GFH & 6.108 & $0.000^{*}$ \\
\hline BANADER & 3.379 & $0.000^{*}$ & INOVEST & 3.701 & $0.000^{*}$ \\
\hline BARKA & 5.468 & $0.000^{*}$ & INVCORP & 0.456 & 0.985 \\
\hline BASREC & 2.060 & $0.000^{*}$ & ITHMR & 7.751 & $0.000^{*}$ \\
\hline BATELCO & 7.342 & $0.000^{*}$ & KHCB & 8.222 & $0.000^{*}$ \\
\hline BBK & 6.064 & $0.000^{*}$ & NASS & 7.215 & $0.000^{*}$ \\
\hline BCFC & 4.303 & $0.000^{*}$ & NBB & 5.226 & $0.000^{*}$ \\
\hline BFM & 2.840 & $0.000^{*}$ & NHOTEL & 2.467 & $0.000^{*}$ \\
\hline BHOTEL & 1.774 & $0.004^{*}$ & POLTRY & 1.447 & $0.030^{*}$ \\
\hline BISB & 4.992 & $0.000^{*}$ & SALAM & 6.996 & $0.000^{*}$ \\
\hline BKIC & 2.703 & $0.000^{*}$ & SEEF & 7.578 & $0.000^{*}$ \\
\hline BMB & 0.872 & 0.433 & TRAFCO & 3.401 & $0.000^{*}$ \\
\hline BMMI & 4.192 & $0.000^{*}$ & UFC & 0.368 & 0.999 \\
\hline $\mathrm{BHN}$ & 0.846 & 0.471 & UGB & 2.440 & $0.000^{*}$ \\
\hline BTC & 2.894 & $0.000^{*}$ & UGIC & 2.653 & $0.000^{*}$ \\
\hline CINEMA & 2.378 & $0.000^{*}$ & ZAINABH & 2.124 & $0.000 *$ \\
\hline
\end{tabular}

Note: ${ }^{*}$ significant at 0.05 level.

Table 2 presents the results of KolmogorovSmirnov goodness of fit test (K-S test). It provides evidence whether or not the distribution confirms to a normal distribution. Results for 40 out of 43 companies are available. The K-S test result shows 0.000 significance at 0.05 level for 36 out of 43 stocks. This indicates that the frequency distribution of the daily prices of the shares does not fit normal distribution, thereby rejecting the null hypothesis and concluding that the share price movements do not follow random walk model according to the K-S tests (refer to Ha1). The results for GLOBAL, TAKAFUL and UPI are not arrived at due to lack of data.

Table 3 presents the results of the run test for the companies listed in the Bahrain Stock Exchange.
The p-values for Arab Banking Corporation (ABC), Aluminum Bahrain B.S.C (ALBH), Al Baraka Banking Group (BARKA), GFH Financial Group (GFH), INOVEST, National Bank of Bahrain (NBB) and Al Salam Bank - Bahrain B.S.C (SALAM) is less than 0.05 and hence reject the null hypothesis. It can be concluded that share prices of these companies do not follow random walk (refer to Ha2). The results for GLOBAL, TAKAFUL and UPI are not arrived at due to lack of data.

From Table 4, it can be observed that results are obtained for 39 of 43 companies. The share prices exhibit low to moderate correlation. The results for the individual companies are mixed, varying from negative to positive values. Ahli United 
Table 3. Results of run test

Source: authors' calculations.

\begin{tabular}{|c|c|c|c|c|c|c|c|}
\hline Company & Test value & $\begin{array}{c}\text { Cases }< \\
\text { Test value }\end{array}$ & $\underset{\text { Test value }}{\text { Cases }} \geq$ & $\begin{array}{l}\text { Total } \\
\text { cases }\end{array}$ & $\begin{array}{l}\text { Number } \\
\text { of runs }\end{array}$ & Z-value & p-value \\
\hline$A B C$ & 0.0239 & 135 & 47 & 182 & 60 & -2.084 & $0.037^{*}$ \\
\hline AHLIA & -0.6468 & 19 & 60 & 79 & 31 & 0.3548 & 0.723 \\
\hline ALBH & -0.0710 & 215 & 593 & 808 & 340 & 2.1114 & $0.035^{*}$ \\
\hline ARIG & -0.0305 & 48 & 172 & 220 & 70 & -1.202 & 0.229 \\
\hline$A \cup B$ & 0.0631 & 660 & 296 & 956 & 404 & -0.432 & 0.666 \\
\hline BANADER & -0.2128 & 9 & 61 & 70 & 15 & -0.923 & 0.356 \\
\hline BARKA & -0.1618 & 131 & 342 & 473 & 208 & 2.0195 & $0.043^{*}$ \\
\hline BASREC & 0.2800 & 44 & 15 & 59 & 21 & -0.826 & 0.409 \\
\hline BATELCO & -0.0572 & 300 & 714 & 1014 & 423 & -0.037 & 0.971 \\
\hline BBK & 0.0274 & 380 & 171 & 551 & 229 & -0.783 & 0.433 \\
\hline BCFC & 0.1923 & 177 & 91 & 268 & 122 & 0.109 & 0.913 \\
\hline BFM & -0.1125 & 5 & 43 & 48 & 11 & 0.4398 & 0.66 \\
\hline BHOTEL & 0.2222 & 75 & 42 & 117 & 59 & 0.8387 & 0.402 \\
\hline BISB & -0.0682 & 109 & 287 & 396 & 171 & 1.5149 & 0.13 \\
\hline BKIC & 0.1381 & 56 & 13 & 69 & 21 & -0.441 & 0.659 \\
\hline BMB & -3.4236 & 11 & 14 & 25 & 9 & -1.585 & 0.113 \\
\hline BMMI & 0.0729 & 228 & 98 & 326 & 134 & -0.539 & 0.59 \\
\hline $\mathrm{BHN}$ & -0.0626 & 42 & 58 & 100 & 51 & 0.2641 & 0.792 \\
\hline BTC & -0.3049 & 33 & 88 & 121 & 49 & 0 & 1 \\
\hline CINEMA & 0.8856 & 52 & 27 & 79 & 33 & -0.893 & 0.372 \\
\hline CPARK & 0.0736 & 44 & 22 & 66 & 32 & 0.4661 & 0.641 \\
\hline DUTYF & 0.0215 & 262 & 125 & 387 & 159 & -1.31 & 0.19 \\
\hline ESTERAD & -0.1034 & 82 & 277 & 359 & 125 & -0.381 & 0.703 \\
\hline FAMILY & 0.0252 & 24 & 10 & 34 & 15 & 0 & 1 \\
\hline GFH & -0.0855 & 117 & 357 & 474 & 159 & -2.257 & $0.024^{*}$ \\
\hline INOVEST & -0.5467 & 53 & 142 & 195 & 65 & -2.396 & $0.017^{*}$ \\
\hline INVCORP & -18.125 & 2 & 4 & 6 & 4 & 0 & 1 \\
\hline ITHMR & -0.0431 & 205 & 637 & 842 & 329 & 1.6689 & 0.095 \\
\hline KHCB & -0.1469 & 173 & 580 & 753 & 277 & 0.9786 & 0.328 \\
\hline NASS & -0.0455 & 232 & 641 & 873 & 345 & 0.2872 & 0.774 \\
\hline NBB & 0.0633 & 439 & 192 & 631 & 295 & 2.5267 & $0.012^{*}$ \\
\hline NHOTEL & -1.2325 & 6 & 26 & 32 & 11 & 0 & 1 \\
\hline POLTRY & 1.5288 & 17 & 9 & 26 & 9 & -1.452 & 0.147 \\
\hline SALAM & 0.0347 & 709 & 279 & 988 & 365 & -2.862 & $0.004^{*}$ \\
\hline SEEF & 0.0394 & 514 & 158 & 672 & 230 & -1.364 & 0.173 \\
\hline TRAFCO & -0.1975 & 27 & 95 & 122 & 41 & -0.543 & 0.587 \\
\hline UFC & 3.900 & 1 & 1 & 2 & 2 & 0 & 1 \\
\hline UGB & -0.0200 & 37 & 92 & 129 & 53 & -0.168 & 0.867 \\
\hline UGIC & 0.0194 & 80 & 38 & 118 & 45 & -1.595 & 0.111 \\
\hline UPI & 0 & 0 & 5 & 5 & 1 & & \\
\hline ZAINABH & -0.0329 & 5 & 26 & 31 & 9 & 0 & 1 \\
\hline
\end{tabular}

Note: ${ }^{*}$ significant at 0.05 level. 
Source: authors' calculations.

\begin{tabular}{|c|c|c|c|c|c|c|c|c|c|c|c|c|c|c|c|c|}
\hline$\stackrel{\text { Lag }}{\text { Company }}$ & 1 & 2 & 3 & 4 & 5 & 6 & 7 & 8 & 9 & 10 & 11 & 12 & 13 & 14 & 15 & 16 \\
\hline $\mathrm{ABC}$ & 0.104 & 0.063 & 0.079 & -0.016 & -0.051 & 0.001 & 0.008 & -0.032 & 0.044 & 0.014 & 0.069 & -0.023 & 0.027 & 0.050 & 0.006 & -0.010 \\
\hline AHLIA & -0.072 & -0.209 & -0.053 & -0.169 & 0.018 & 0.139 & -0.030 & -0.075 & 0.010 & 0.036 & -0.126 & 0.081 & 0.019 & -0.005 & 0.143 & -0.070 \\
\hline ALBH & -0.055 & 0.051 & $0.105^{*}$ & $0.055^{*}$ & $0.059^{*}$ & $-0.018^{*}$ & $0.124^{*}$ & $0.099 *$ & $0.028^{*}$ & $-0.064^{*}$ & 0.013* & $0.062^{*}$ & $-0.039 *$ & $0.008^{*}$ & $0.001^{*}$ & $-0.042^{*}$ \\
\hline ARIG & 0.018 & -0.113 & -0.042 & -0.093 & 0.047 & -0.072 & -0.048 & 0.108 & -0.041 & 0.091 & 0.003 & -0.034 & 0.014 & 0.001 & -0.011 & -0.057 \\
\hline AUB & $-0.064^{*}$ & $-0.125^{*}$ & $-0.020^{*}$ & $-0.033^{*}$ & $0.060^{*}$ & $0.035^{*}$ & $-0.014^{*}$ & $-0.040^{*}$ & $-0.009^{*}$ & $0.032 *$ & $0.029 *$ & $0.005^{*}$ & $-0.032^{*}$ & $-0.035^{*}$ & $-0.018^{*}$ & $0.070^{*}$ \\
\hline BANADER & 0.142 & -0.216 & 0.109 & 0.120 & -0.083 & -0.002 & -0.053 & -0.125 & 0.092 & 0.079 & -0.001 & 0.070 & 0.122 & -0.004 & -0.092 & -0.021 \\
\hline BARKA & $-0.102^{*}$ & $-0.075^{*}$ & $-0.051^{*}$ & $-0.065^{*}$ & 0.019 & -0.012 & 0.036 & -0.064 & -0.039 & 0.013 & $-0.114^{*}$ & $0.080^{*}$ & $0.004^{*}$ & $-0.039 *$ & $-0.056^{*}$ & $0.032^{*}$ \\
\hline BASREC & -0.075 & 0.049 & -0.036 & 0.119 & -0.057 & -0.046 & -0.208 & -0.099 & -0.055 & -0.027 & 0.117 & 0.073 & -0.109 & 0.051 & 0.111 & 0.007 \\
\hline BATELCO & -0.041 & -0.011 & 0.061 & -0.006 & 0.017 & 0.042 & -0.008 & -0.028 & 0.009 & -0.054 & 0.009 & -0.055 & -0.048 & 0.052 & 0.047 & $-0.086^{*}$ \\
\hline BBK & $-0.146^{*}$ & $-0.108^{*}$ & $0.003^{*}$ & $0.028^{*}$ & $-0.029^{*}$ & $-0.034^{*}$ & $0.036^{*}$ & $0.034^{*}$ & $-0.027^{*}$ & $-0.023^{*}$ & $0.002^{*}$ & $-0.022^{*}$ & $-0.020^{*}$ & -0.004 & 0.029 & $-0.098^{*}$ \\
\hline BCFC & $-0.145^{*}$ & $-0.076^{*}$ & $-0.109^{*}$ & $0.129 *$ & $-0.008^{*}$ & $0.008^{*}$ & $-0.021^{*}$ & 0.027 & 0.002 & -0.066 & 0.095 & $-0.162^{*}$ & $0.132 *$ & $-0.019^{*}$ & $0.106^{*}$ & $-0.035^{*}$ \\
\hline BFM & $-0.314^{*}$ & $0.085^{*}$ & -0.002 & -0.141 & 0.242 & -0.068 & 0.163 & -0.145 & 0.117 & 0.066 & -0.003 & -0.004 & 0.056 & 0.026 & -0.063 & 0.004 \\
\hline BHOTEL & -0.094 & 0.077 & 0.106 & -0.142 & -0.044 & 0.065 & 0.095 & -0.124 & 0.150 & -0.073 & -0.056 & 0.002 & 0.018 & -0.112 & -0.023 & -0.030 \\
\hline BISB & 0.009 & 0.001 & 0.055 & 0.036 & 0.093 & -0.049 & 0.005 & -0.019 & -0.002 & 0.018 & -0.098 & -0.016 & 0.036 & -0.027 & 0.012 & -0.018 \\
\hline BKIC & 0.117 & -0.053 & 0.063 & 0.051 & 0.043 & -0.069 & -0.050 & -0.175 & -0.145 & 0.077 & 0.031 & -0.010 & 0.036 & 0.050 & 0.209 & -0.069 \\
\hline $\mathrm{BMB}$ & 0.132 & -0.234 & $-0.484^{*}$ & $-0.159^{*}$ & -0.025 & $0.338^{*}$ & $0.099^{*}$ & -0.074 & -0.044 & -0.097 & -0.136 & 0.025 & 0.184 & 0.078 & 0.038 & 0.009 \\
\hline BMMI & 0.032 & -0.034 & -0.017 & 0.021 & 0.078 & 0.025 & -0.075 & -0.038 & 0.010 & -0.040 & 0.048 & 0.001 & -0.031 & 0.010 & 0.007 & -0.007 \\
\hline $\mathrm{BHN}$ & -0.131 & -0.076 & -0.074 & 0.085 & 0.019 & -0.017 & -0.025 & -0.036 & 0.046 & -0.145 & 0.015 & -0.068 & -0.056 & 0.098 & -0.062 & -0.054 \\
\hline BTC & 0.070 & 0.007 & -0.010 & 0.093 & -0.075 & -0.186 & -0.021 & -0.070 & -0.007 & -0.064 & -0.018 & -0.144 & -0.084 & -0.052 & 0.137 & -0.077 \\
\hline CINEMA & -0.031 & -0.016 & 0.094 & -0.010 & 0.049 & -0.070 & -0.100 & -0.223 & 0.040 & -0.143 & -0.035 & -0.143 & 0.075 & 0.000 & 0.042 & 0.148 \\
\hline
\end{tabular}


Table 4 (cont). Results of autocorrelation of individual stocks

\begin{tabular}{|c|c|c|c|c|c|c|c|c|c|c|c|c|c|c|c|c|}
\hline $\begin{array}{l}\text { Lag } \\
\text { Company }\end{array}$ & 1 & 2 & 3 & 4 & 5 & 6 & 7 & 8 & 9 & 10 & 11 & 12 & 13 & 14 & 15 & 16 \\
\hline CPARK & 0.009 & -0.084 & 0.186 & -0.091 & 0.031 & 0.187 & -0.181 & -0.163 & -0.004 & -0.003 & -0.167 & 0.097 & -0.089 & -0.169 & 0.089 & 0.019 \\
\hline DUTYF & $-0.173^{*}$ & $0.074^{*}$ & $0.019^{*}$ & $-0.002^{*}$ & $-0.007^{*}$ & $0.084^{*}$ & $0.003^{*}$ & $-0.008^{*}$ & -0.002 & -0.002 & -0.045 & 0.023 & 0.017 & -0.016 & -0.070 & -0.020 \\
\hline ESTERAD & -0.012 & 0.067 & 0.125 & $0.089^{*}$ & $0.146^{*}$ & $0.011^{*}$ & $0.009 *$ & $0.071^{*}$ & $-0.024^{*}$ & $0.044^{*}$ & $0.137^{*}$ & $-0.054^{*}$ & $0.067^{*}$ & $-0.039^{*}$ & $-0.010^{*}$ & $0.016^{*}$ \\
\hline FAMILY & -0.042 & -0.245 & 0.053 & -0.148 & 0.141 & 0.251 & -0.341 & -0.280 & $0.306^{*}$ & $-0.003^{*}$ & $0.037^{*}$ & $0.155^{*}$ & $-0.175^{*}$ & $0.029^{*}$ & $0.161^{*}$ & $-0.188^{*}$ \\
\hline GFH & 0.017 & -0.015 & 0.042 & 0.080 & $-0.145^{*}$ & $0.000^{*}$ & $0.021^{*}$ & $0.054^{*}$ & $-0.169^{*}$ & $-0.041^{*}$ & $-0.008^{*}$ & $0.005^{*}$ & $-0.048^{*}$ & $-0.082^{*}$ & $-0.025^{*}$ & $-0.005^{*}$ \\
\hline INOVEST & 0.000 & -0.022 & -0.042 & 0.130 & 0.008 & 0.038 & 0.041 & -0.067 & 0.028 & 0.018 & 0.067 & -0.036 & 0.031 & 0.104 & -0.105 & 0.010 \\
\hline INVCORP & 0.025 & -0.138 & -0.126 & -0.236 & - & - & - & - & - & - & - & - & - & - & - & - \\
\hline ITHMR & 0.049 & 0.059 & 0.018 & 0.028 & -0.028 & 0.001 & 0.016 & 0.053 & -0.003 & 0.007 & 0.031 & -0.016 & -0.032 & -0.043 & -0.028 & 0.000 \\
\hline $\mathrm{KHCB}$ & 0.036 & 0.020 & $0.099^{*}$ & $-0.047^{*}$ & $0.059^{*}$ & $0.022^{*}$ & -0.010 & 0.034 & 0.036 & -0.040 & -0.039 & -0.045 & $-0.071^{*}$ & -0.025 & 0.018 & 0.013 \\
\hline NASS & 0.013 & -0.013 & 0.052 & -0.033 & $-0.097^{*}$ & $-0.094^{*}$ & $-0.051^{*}$ & $-0.014^{*}$ & $0.022^{*}$ & $0.009^{*}$ & $0.019^{*}$ & $0.025^{*}$ & $0.051^{*}$ & $0.007^{*}$ & $-0.033^{*}$ & $-0.002^{*}$ \\
\hline NBB & $-0.213^{*}$ & $-0.094^{*}$ & $0.121^{*}$ & $0.011^{*}$ & $-0.048^{*}$ & $0.073^{*}$ & $-0.025^{*}$ & $-0.004^{*}$ & $0.042^{*}$ & $-0.009^{*}$ & $-0.051^{*}$ & $0.054^{*}$ & $0.026^{*}$ & $-0.006^{*}$ & $-0.008^{*}$ & $-0.087^{*}$ \\
\hline NHOTEL & -0.087 & -0.112 & 0.102 & -0.239 & 0.058 & 0.333 & -0.319 & -0.012 & 0.063 & -0.080 & 0.113 & 0.116 & -0.265 & 0.005 & 0.066 & -0.053 \\
\hline POLTRY & 0.098 & 0.133 & -0.038 & -0.138 & 0.052 & -0.076 & 0.048 & -0.268 & -0.101 & 0.068 & -0.163 & -0.113 & 0.008 & -0.032 & 0.137 & 0.068 \\
\hline SALAM & $0.115^{*}$ & $0.036^{*}$ & $-0.027^{*}$ & $0.075^{*}$ & $0.021^{*}$ & $-0.008^{*}$ & $0.041^{*}$ & $0.065^{*}$ & $0.043^{*}$ & $0.011 *$ & $-0.061^{*}$ & $-0.006^{*}$ & $0.010^{*}$ & $-0.010^{*}$ & $0.001^{*}$ & $-0.026^{*}$ \\
\hline SEEF & $-0.096^{*}$ & $0.101^{*}$ & $0.011^{*}$ & $0.081^{*}$ & $0.036^{*}$ & $-0.022^{*}$ & $0.053^{*}$ & $0.006^{*}$ & $-0.002^{*}$ & $0.012^{*}$ & $0.077^{*}$ & $0.011^{*}$ & $0.024^{*}$ & $-0.048^{*}$ & $0.033^{*}$ & $0.017^{*}$ \\
\hline TRAFCO & 0.044 & -0.109 & -0.052 & -0.051 & -0.042 & 0.008 & -0.086 & -0.005 & 0.039 & -0.016 & 0.109 & 0.141 & -0.080 & -0.149 & -0.143 & 0.002 \\
\hline UGB & $0.213^{*}$ & $0.228^{*}$ & $0.135^{*}$ & $0.085^{*}$ & $0.018^{*}$ & $0.065^{*}$ & $-0.060^{*}$ & $-0.086^{*}$ & $0.128^{*}$ & $-0.174 *$ & $0.021^{*}$ & $-0.021^{*}$ & $-0.140^{*}$ & $0.043^{*}$ & $-0.035^{*}$ & $-0.129 *$ \\
\hline UGIC & 0.132 & $0.188^{*}$ & 0.002 & 0.024 & -0.050 & -0.081 & -0.203 & $-0.195^{*}$ & $-0.143^{*}$ & $-0.097^{*}$ & $-0.132 *$ & $-0.203^{*}$ & $-0.041^{*}$ & $-0.096^{*}$ & $0.175^{*}$ & $-0.023^{*}$ \\
\hline ZAINABH & -0.004 & -0.184 & 0.088 & -0.187 & -0.185 & 0.087 & 0.176 & -0.099 & -0.188 & 0.088 & -0.002 & 0.093 & 0.095 & 0.090 & -0.001 & -0.183 \\
\hline
\end{tabular}


bank (AUB), Bank of Bahrain and Kuwait (BBK), National Bank of Bahrain (NBB), Al Salam Bank - Bahrain B.S.C (SALAM), Seef Properties B.S.C (SEEF), United Gulf bank (UGB) have significant correlation values throughout the 16 lags. Aluminum Bahrain B.S.C (ALBH), Esterad Investment Co. (ESTERAD), GFH Financial group (GFH), NASS Corporation (NASS), Khaleeji Commercial Bank (KHCB) exhibit significant correlation values from lag 3 onwards. Share price movements of Al Baraka Banking
Group (BARKA), Bahrain Commercial Facilities Co. (BCFC), Bahrain Duty Free Shop Complex Co. (DUTYF), Bahrain Family Leisure Co. (FAMILY) and United Gulf Investment Corporation (UGIC) exhibit significant correlation during some lags. Investcorp Bank (INVCORP) presents correlation only for few lags due to insufficiency of data. Only 6 of 39 companies show significant correlation values, hence, in general, the null hypothesis is rejected. It can be concluded that price changes in individual stocks are not independent (refer to Ha3).

\section{CONCLUSION}

The present study aims to test the weak form of efficiency of the individual stock listed on Bahrain Bourse for the period 2011 to 2015. Delmon Poultry Co. (POLTRY) records the highest mean returns of $1.528 \%$, while the Bahrain All Share Index records a mean return of $-0.013 \%$. The K-S test result conS cludes that in general, the individual stock returns do not follow random walk. Run test shows that the successive price changes are not random (Elango \& Hussein, 2007).

Autocorrelation tests reveal that share prices exhibit low to moderate correlation varying from negative to positive values. The results of the tests do not support the randomness of the returns of the individual stocks. Therefore, it is difficult to conclude that successive returns of the stocks listed on Bahrain Bourse follow a random walk. These conclusions are similar to Solnik (1973), Rao and Shankaraiah (2003), Moustafa (2004), and Elango and Hussein (2007).

\section{REFERENCES}

1. Abeysekera, S. P. (2001). Efficient Markets Hypothesis and the Emerging Capital market in Sri Lanka: Evidence from the Colombo Stock Exchange - A Note. Journal of Business Finance and Accounting, 28(1), 249-261.

2. Abraham, A., Seyyed, F. J., \& Alsakran, S. A. (2002). Testing the Random Walk Behavior and Efficiency of the Gulf Stock Markets. The Financial Review, 37(3), 469-480.

3. Ananzeh, I. E. (2014). Testing the Weak Form of Efficient Market Hypothesis: Empirical Evidence from Jordan. International Business and Management, 9(2), 119-123.

4. Ang, J., \& Pohlman, A. R. (1978). A note on the price behaviors of Far Eastern Stocks. Journal of International Business Studies, 103-107.
5. Asiri, B. K. (2008). Testing Weak from Efficiency in the Bahrain Stock Market. International Journal of Emerging Markets, 3(1), 38-55.

6. Awan, U. F., \& Subayyal, M. (2016, June 2). Social Science Research Network. Retrieved December 19, 2016 from Social Science Research Network https://papers.ssrn. $\mathrm{com} / \mathrm{sol} 3 /$ papers2.cfm?abstract $\mathrm{id}=2787816$

7. Butler, K. C., \& Malaikah, S. J. (1992). Efficiency and inefficiency in thinly traded stock markets, Kuwait and Saudi Arabia. Journal of Banking and Finance, 16, 197-210.

8. Chan, K. C., Gup, B. E., \& Pan, M.S. (1992). An Empirical Analysis of Stock Prices in Major Asian Markets and the United States. The Financial Review, 27(2), 289-307.

9. Dahel, R., \& Laabas, B. (1999). The Behaviour of Stock Prices in the GCC Markets. Journal of
Development \& Economic Policies, 1, 89-105.

10. Elango, R., \& Hussein, M. I. (2007, November 5). An Empirical Analysis on the Weak-Form Efficiency of the GCC Markets Applying Selected Statistical Tests. Retrieved January 19, 2017 from Social Science Research Network https://papers.ssrn.com/sol3/papers.cfm?abstract_id=1026569

11. Fama, E. F. (1970). Efficient capital markets: A review of theory and empirical work. The Journal of Finance, 25(2), 383-417.

12. Fawson, C., Glover, T. F., Fang, W., \& Chang, T. (1996). The WeakForm Efficiency of the Taiwan Share Market. Applied Economics Letters, 3, 663-667.

13. Groenewold, N., Sam, T., \& Wu, Y. (2003). The Efficiency of the Chinese Stock Market and the Role of the Banks. Journal of Asian Economics, 14, 593-609. 
14. Hawaldar, I. T., Shakila, B., \& Pinto, P. (2017). Empirical Testing of Month of the Year Effect on Selected Commercial Banks and Services Sector Companies Listed on Bahrain Bourse. International Journal of Economics and Financial Issues, 7(2), 426-436. Retrieved from https://www.econjournals.com/index.php/ijefi/article/view/4131/pdf

15. Hawaldar, I. T. (2016b). The CrossSectional Variations in Portfolio Returns: Evidence from Bahrain Bourse. British Journal of Economics, Finance and Management Sciences, 12(2), 1-11. Retrieved from http:// www.ajournal.co.uk/EFpdfs/EFvolume12(2)/EFVol.12\%20(2)\%20 Article\%201.pdf

16. Hawaldar, I. T. (2016a). The Reaction of Bahrain Bourse to Announcement of Annual Financial Results. International Review of Business Research Papers, 12(1), 64-75. http:// dx.doi.org/10.21102/ irbrp.2016.03.121.05. Retrieved from https://zantworldpress.com/productsingle/?poId=2238\&pageId $=2224$

17. Hawaldar, I. T. (2015). Empirical Testing of Capital Asset Pricing Model on Bahrain Bourse. Asian Journal of Finance \& Accounting, 7(2), 107-119. https://doi. org/10.5296/ajfa.v7i2.8356
18. Iqbal, \& Mallikarjunappa, T. (2011). Efficiency of Stock Market: A Study of Stock Price Responses to Earnings Announcements. LAP Lambert Academic Publishing Company, Germany.

19. Iqbal, \& Mallikarjunappa, T. (2010). A Study of Efficiency of the Indian Stock Market. Indian Journal of Finance, 4(5), 32-38. Retrieved from http://www.indianjournaloffinance.co.in/index.php/IJF/article/ view/72610

20. Iqbal, \& Mallikarjunappa, T. (2008). The Behavior of Indian Stock Prices and Returns: Is the Stock Market Efficient? Scour, 2(2), 39-46. Retrieved from https:// www.researchgate.net/publication/316665979_The_Behaviour_of_ Indian Stock Prices and Returns Is_the_Stock_Market_Efficient

21. Karemera, D., Ojah, K., \& Cole, J. A. (1999). Random Walks and Market Efficiency Tests: Evidence from Emerging Equity Markets. Review of Quantitative Finance and Accounting, $13,171-188$.

22. Lo, A. W., \& MacKinlay, A. C. (1988). Stock market prices do not follow random walks: Evidence from a simple specification test. The Review of Financial Studies, 1(1), 41-66.

23. Mookerjee, R., \& Yu, Q. (1999). An Empirical Analysis of the
Equity Markets in China. Review of Financial Economics, 8, 41-60.

24. Moustafa, M. A. (2004). Testing the Weak-Form Efficiency of the United Arab Emirates Stock Market. International Journal of Business, 9(3), 309-325.

25. Rao, D. N., \& Shankaraiah, K. (2003). Stock Market Efficiency and Strategies for Developing GCC Financial Markets: A Case Study of the Bahrain Stock Market. The Arab Bank Review, 5(2), 16-21.

26. Seddighi, H. R., \& Nian, W. (2004). The Chinese Stock Exchange Market: Operations and Efficiency. Applied Financial Economics, 14, 785-797.

27. Sharma, J. L., \& Kennedy, R. E. (1977). A comparative Analysis of Stock Price Behavior on the Bombay, London, and New York Stock Exchanges. Journal of Financial and Quantitative Analysis, 391-413.

28. Sharma, S. N. (2005). On the nonnormality of GCC Stock Markets. The ICFAI Journal of Applied Corporate Finance, 51-58.

29. Solnik, B. (1973). Note on the validity of the random walk for European stock prices. Journal of Finance, 28(5), 1151-1159. 